Objectives To evaluate the correlation between with mammographically detected 4 BI-RAD microcalcifications and histopathological findings in patientsto allow a better surgical planning.

Methods Eighteen patients who had a 4 BI-RADS micocalcifications on mammography were admitted to our hospital were analyzed retrospectively.

Results Our study included nine patients; all patients were women, Breastfeeding was noted in eleven patients. Physical examination was negative in all patients. The mammography showed microcalcification in 17 cases, mass and microcalcifications in one case, it was localized on the upper outer quadrant of the breast in the majority of cases (12 cases). It had regional distribution in 10 cases, multiple in 4 cases, linear in the lumen ( 2 cases) and polymorphous microcalcifications in 13 cases. It was classified on BI-RADS 4 A (5 cases), BI-RADS 4B ( 9 cases) and BI-RADS $4 \mathrm{C}$ in 4 cases. One patient underwent a core needle biopsy, two patients had a macrobiopsy (VAB System) and 17 patients underwent a surgical excision in all cases. The histological examination revealed a ductal carcinoma in situ (2 cases), invasive ductal carcinoma with ductal carcinoma in situ (1case) and and benign lesions in 15 cases. Our study did not found a correlation between BIRADs classification and histological finding because of the shortage of the study.

Conclusions Microcalcifications are actually indirect signs of pathological processes, some of which may only be correctly identified according to their morphology. This is true for the microcalcifications classified as typically benign in the 4th edition of the BI-RADS system.

\section{EPV033/\#626 THE IMPACT OF THE COVID-19 PANDEMIC ON BREAST CANCER PATIENTS AWAITING SURGERY: THE EXPERIENCE OF SALAH AZAIEZ INSTITUTE TUNISIA}

M Bouhani*, S Sakhri, A Jerbi, MA Ayedi, R Chargui, K Rahal. Salah Azaiez Institute of Oncology, Surgical Oncology, Tunis, Tunisia

10.1136/ijgc-2021-IGCS. 100

Objectives The aim of our study is to study the impact of covid19 on patients waiting surgery.

Methods We retrospectively reported 33 patients diagnosed breast carcinoma and underwent surgery at salah azaiez institute Tunisia between 18/3/2020 and 29/3/2020 (72 days).

Results During the first pandemic of COVID-19, 33 patients underwent breast surgery, the mean age was 51 years (ranging 34-82). 28 patients had Social insurance. 28 patients patient belongs to urban environment.11 patients had neoadjuvant chemotherapy.Invasive ductal carcinoma is more frequent (30 cases), tumors had a high brad nuclear in 18 cases. In 16 cases the tumor had stade IIB, axillary lymph node metastasis were found in 20 patients. 11 patients underwent radical surgery and only one patient had plastic reconstruction.

Conclusions The COVID-19 pandemic has affected just about every aspect of life, including screening, diagnosis, treatment, and follow-up care for breast cancer. People who've been diagnosed with breast cancer and people who are at high risk for breast cancer have found themselves in a uniquely difficult and sometimes frightening position since the coronavirus crisis began.

\section{EPV033a/\#697 SIGNIFICANCE OF HISTOLOGY AND NODAL STATUS ON THE SURVIVAL OF WOMEN WITH EARLY-STAGE CERVICAL CANCER: VALIDATION OF THE 2018 FIGO CERVICAL CANCER STAGING SYSTEM}

H Machida*, K Matsuo, Y Kobayashi, M Momomura, F Takahashi, T Tabata, E Kondo, W Yamagami, Y Ebina, M Kaneuchi, S Nagase, M Mikami. Tokai University School of Medicine: Tokai Daigaku Igakubu Daigakuin Igaku Kenkyuka, Obstetrics and Gynecology, Kanagawa, Japan

\subsection{6/ijgc-2021-IGCS.101}

Objectives To examine the prognostic impact of a node-specific staging system for stage IB cervical cancer based on the 2018 FIGO classifications and to assess the efficacy of postoperative adjuvant therapy for nodal metastasis in stage IIIC cervical cancer.

Methods This is a society-based retrospective observational study in Japan, examining 16,539 women with stage IB1 cervical cancer who underwent primary surgical treatment from 2004-2015. Associations between nodal metastasis and causespecific survival (CSS) and postoperative adjuvant therapy and CSS were examined according to histology type (Squamous cell carcinoma [SCC] $n=10,315$ and non-SCC $n=6,224)$.

Results The nodal metastasis rate for SCC was higher than that for non-SCC $(10.7 \%$ versus $8.3 \%, \mathrm{P}<0.001)$. In a multivariable analysis, the impact of pelvic nodal metastasis on CSS for non-SCC tumors (adjusted-hazard ratio [HR] 2.89, 95\% confidence interval [CI] 1.93-4.31) was larger than for SCC tumors (adjusted-HR, 1.84, 95\%CI 1.38-2.44). A propensity score matching analysis showed that women with pelvic nodal metastases had significantly lower CSS rates with non-SCC tumors than with SCC tumors (5-year CSS, 75.4\% versus 90.3\%, $\mathrm{P}<0.001)$. Postoperative chemotherapy improved CSS for women with pelvic nodal metastases (HR 0.65, 95\%CI $0.44-0.95, \mathrm{P}=0.024)$; however, the efficacy of postoperative chemotherapy on CSS for these was differ according to histology type.

Conclusions For stage IB1 cervical cancer, the node-specific staging system in the 2018 FIGO cervical cancer classification is more applicable to non-SCC tumors than to SCC tumors. The survival benefits of postoperative adjuvant therapy for IIIC1 patients likely differ between SCC and non-SCC tumors.

\section{EPV034/\#116 PREDICTING THE RATE OF ADJUVANT POSTOPERATIVE CHEMO/RADIATION OF PATIENTS WITH THE RECENTLY UPDATED STAGE IB2 CERVICAL CANCER: AN ISRAELI GYNECOLOGIC ONCOLOGY GROUP STUDY}

${ }^{1} 0$ Gemer* ${ }^{1}$ A Namazov, ${ }^{2} \mathrm{~A}$ Ben Arie, ${ }^{3} \mathrm{R}$ Eitan, ${ }^{4} \mathrm{~A}$ Rabinovich, ${ }^{5} \mathrm{Z}$ Vaknin, ${ }^{6} \mathrm{~S}$ Armon ${ }^{7}$ I Bruchim, ${ }^{8} \mathrm{~T}$ Levy, ${ }^{9}$ I Ben Shachar, ${ }^{10} \mathrm{O}$ Lavie. ${ }^{1}$ Barzilai Medical Center, Gynecology, Ashkelon, Israel; ${ }^{2}$ Kaplan Medical Center, Hebrew University, Gynecology, Rehovot, Israel; ${ }^{3}$ Rabin Medical Center, Gynecology, Petah Tikva, Israel; ${ }^{4}$ Soroka Medical Center, Gynecology, Beer Sheva, Israel; ${ }^{5}$ Assaf Haroffe Medical Center, Sackler School of Medicine, Gynecology, Zrifin, Israel; '5Shaare Zedek Medical Center, Hebrew University, Gynecology, Jerusalem, Israel; ' Meir Medical Center, Sackler School of Medicine, Tel Aviv University, Gynecology, Kfar Saba, Israel; ${ }^{8}$ Wolfson Medical Center, Holon, Sackler Faculty of Medicine, Tel Aviv University, Gynecology, Tel Aviv, Israel; ${ }^{9}$ Hadassah Medical Center, Hebrew University, Gynecology, Jerusalem, Israel; ${ }^{10}$ Carmel Medical Center, Obstetrics and Gynecology, Haifa, Israel

\subsection{6/ijgc-2021-IGCS.102}


Objectives Women with cervical cancer who undergo radical hysterectomy are often treated postoperatively with chemoradiation. The patient selection that minimizes adjuvant treatment is valuable. We compared two methods for predicting postoperative adjuvant treatment of patients with stage IB2 cervical cancer.

Methods This multicenter retrospective study included 272 women with IB2 tumors. A receiver operating characteristic curve (ROC) analysis was used to determine the optimal tumor cutoff size to predict adjuvant treatment. A second analysis compared the rate of adjuvant treatment between women with and without lymph vascular space involvement (LVSI).

Results According to the ROC, the optimal cutoff value of tumor size for predicting adjuvant treatment was $2.95 \mathrm{~cm}$ (sensitivity 0.70 , specificity 0.67 ). Tumors were $\geq 3.0 \mathrm{~cm}$ in $166(61.0 \%)$ women. The rate of adjuvant treatment was higher in women with larger tumor diameter $(73.8 \%$ vs. $47.9 \%, \mathrm{p}<0.0001)$. of the 241 women with a LVSI record, LVSI was present in 81 (34\%) women. Among women with LVSI, rates were higher of positive lymph nodes $(41.0 \%$ vs $14.5 \%, \quad \mathrm{p}<0.0001)$ and postoperative adjuvant treatment $(83.3 \%$ vs. $53.7 \%, \mathrm{p}<0.001)$. Among women with tumor size $\geq 3.0 \mathrm{~cm}$ and LVSI, the rate of adjuvant treatment was $90.0 \%$. In the multivariate analysis, both tumor size $\geq 3.0 \mathrm{~cm}$ and the presence of LVSI were independently associated with adjuvant treatment (OR 3.9, 95\% CI 2.1-7.1; p <0.0001 and OR 4.9, 95\% CI 2.4-10.0; $\mathrm{p}<0.0001$, respectively).

Conclusions These data should be weighed in multidisciplinary consultation with radiation oncologists when deciding treatment strategy.

\section{EPV035/\#138 RADICAL TRACHELECTOMY. EXPERIENCE IN KAZIOR}

${ }^{1}$ A Satanova, ${ }^{2} \mathrm{D}$ Kaidarova*, ${ }^{3} \mathrm{R}$ Bolatbekova, ${ }^{1} \mathrm{Y}$ Kukubassov, ${ }^{1} \mathrm{D}$ Kaldybekov. ${ }^{1}$ Kazakh Institute of Oncology and Radiology, Oncogynecology, Almaty, Kazakhstan; ${ }^{2}$ Kazakh Institute of Oncology and Radiology, Head of Kazior, Almaty, Kazakhstan; ${ }^{3}$ Almaty Oncologycal Center, Oncogynecology, Almaty, Kazakhstan

\subsection{6/ijgc-2021-IGCS.103}

Objectives To investigate pregnancy outcomes in women after radical trachelectomy (RT) in KazIOR for early-stage cervical cancer

Methods Systematic analysis of the data of the cancer register of the Republic of Kazakhstan

Results Since 2013, radical trachelectomy has been performed at KazIOR. From 2013 to 2021, 8 operations were performed, 7 of them by abdominal access, 3 by laparoscopic approach. $6(75 \%)$ of the patients had stage 1 B1 from 2 to $4 \mathrm{~cm} ; 2$ (25\%) had a 1a1 stage.The average age of patients was 28 years (from 26 to 37 years). 5 (62.5\%) patients were nulliparous, 2 patients had 2 children, 1 patient had 1 child.

LVSI was negative in preoperative histological examination, and resection margins were also negative. The histological form of the tumor in all cases was squamous cell carcinoma. On average, 11 lymph nodes were removed. In 1 patient (12.5\%) after histological examination LVSI was positive, in 7 it was negative. None of the patients had metastases to the pelvic lymph nodes. During express histology, the resection margins were negative in all patients. Patients in the postoperative period were not prescribed chemoradiation therapy. of the 8 patients who retained fertility, there were 5 pregnancies, 2 miscarriages at 9-10 weeks, and 3 deliveries at 36-37 weeks of gestation.

Conclusions Thus, in 2013-2021, 8 radical trachelectomy operations were successfully performed. The data presented in this publication demonstrate that patients with stage IB1 tumors ranging in size from 2 to $4 \mathrm{~cm}$ and with favorable histology are acceptable candidates for attempted radical trachelectomy.

\section{EPV036/\#142 EUROPEAN NETWORK FOR GYNAECOLOGICAL ONCOLOGICAL TRIAL (ENGOT)-CX11/ GYNECOLOGIC ONCOLOGY GROUP (GOG) 3047I KEYNOTE-A18: PHASE 3 TRIAL OF PEMBROLIZUMAB PLUS CHEMORADIOTHERAPY IN HIGH-RISK LOCALLY ADVANCED CERVICAL CANCER}

${ }^{1} \mathrm{D}$ Lorusso*, ${ }^{2} Y$ Xiang, ${ }^{3} \mathrm{~N}$ Colombo, ${ }^{4} \mathrm{RL}$ Coleman, ${ }^{5} \mathrm{LM}$ Randall, ${ }^{6} \mathrm{~L}$ Duska, ${ }^{7} \mathrm{~K}$ Hasegawa, ${ }^{8} \mathrm{~A}$ Nogueira-Rodrigues, ${ }^{9} \mathrm{D}$ Cibula, ${ }^{10} \mathrm{M}$ Mirza, ${ }^{11} \mathrm{~B}$ You, ${ }^{12} \mathrm{~A}$ Oaknin, ${ }^{13} \mathrm{M}$ Christiaens, ${ }^{14} \mathrm{C}$ Taskiran, ${ }^{15} \mathrm{~J}$ Sehouli, ${ }^{16} \mathrm{~J}$ Korach, ${ }^{17} \mathrm{C}$ Marth, ${ }^{18} \mathrm{~K}$ Yamada, ${ }^{18} \mathrm{M}$ Puglisi, ${ }^{19} \mathrm{~S}$ Pignata. ${ }^{1}$ Associate Professor of Obstetrics and Gynecology, Catholic University of Sacred Heart, Clinical Research Development Unit Fondazione Policlinico Gemelli Irccs, Rome, Italy; ${ }^{2}$ Peking Union Medical College Hospital, Chinese Academy of Medical Sciences, Department of Gynecology and Obstetrics, Beijing, China; ${ }^{3}$ Instituto Europeo di Oncologia, Department of Gynecology, Milan, Italy; ${ }^{4}$ University of Texas MD anderson Cancer Center, Department of Gynecologic Oncology and Reproductive Medicine, Houston, USA; ${ }^{5}$ Massey Cancer Center, Virginia Commonwealth University, Department of Obstetrics and Gynecology, Richmond, USA; ${ }^{6}$ University of Virginia, Department of Obstetrics and Gynecology, Charlottesville, USA; 'Saitama Medical University, Hidaka, Department of Gynecologic Oncology, Saitama Prefecture, Japan; ${ }^{8}$ Universidade Federal de Minas Gerais, Belo Horizonte, Departamento De Clínica Médica, Minas Gerais, Brazil; ${ }^{9}$ General Faculty Hospital in Prague, First Faculty of Medicine, Charles University, Department of Obstetrics and Gynecology, Prague, Czech Republic; ${ }^{10}$ Copenhagen University Hospital, Department of Oncology, Copenhagen, Denmark; ${ }^{11} \mathrm{CITOHL}, I C-H C L$, Hospices Civils de Lyon, Université Claude Bernard Lyon 1, Department of Medical Oncology, Lyon, France; ${ }^{12}$ Vall d'Hebron Institute of Oncology (VHIO), Hospital Universitari Vall d'Hebron, Vall d'Hebron Barcelona Hospital Campus, Department of Medical Oncology, Barcelona, Spain; ${ }^{13}$ Universitair Ziekenhuis Leuven, Department of Radiation Oncology, Leuven, Belgium; ${ }^{14}$ Professor Koc University School of Medicine and VKV American Hospital, Department of Obstetrics and Gynecology, Division of Gynecologic Oncology and Turkish Society of Gynecologic Oncology, Istanbul, Turkey; ${ }^{15}$ Charité-Universitätsmedizin Berlin, Deparment of Gynecology, Berlin, Germany; ${ }^{16}$ Sheba Medical Center, Gynecology Oncology Department, Ramat Gan, Israel; ${ }^{17}$ Medizinische Universität Innsbruck, Department of Obstetrics and Gynecology, Innsbruck, Austria; ${ }^{18}$ Merck and Co., Inc., Clinical Development, Kenilworth, USA; ${ }^{19}$ Instituto Nazionale Tumori IRCCS Fondazione G Pascale, Department of Uro-gynaecological Oncology, Napoli, Italy

\subsection{6/ijgc-2021-IGCS.104}

Objectives High-risk locally advanced cervical cancer has a poor prognosis. External beam radiotherapy (EBRT) with concurrent chemotherapy followed by brachytherapy is the standard of care. The immunostimulatory activity of pembrolizumab may be enhanced by concurrent chemoradiotherapy (CCRT). Pembrolizumab monotherapy is approved for patients with PD-L1-positive recurrent or metastatic cervical cancer that progressed during or after chemotherapy. The phase 3 ENGOT-cx11/GOG 3047/KEYNOTE-A18 (NCT04221945) study is evaluating pembrolizumab with CCRT in patients with locally advanced cervical cancer.

Methods $\sim 980$ patients with high-risk (FIGO 2014 stage IB2IIB with node-positive disease or stage III-IVA), locally 